\title{
Surgical Management of a Case of Bilateral Auricular Keloids in an Adolescent Girl
}

\section{Priya Jeyaraj ${ }^{* 1}$ and Lt Col Sumeet Sehgal ${ }^{2}$}

${ }^{1}$ Classified Specialist in Oral and Maxillofacial Surgery, Deputy Commandant; Officer in Charge Trauma and Rehabilitation, Command Military Dental Centre (Northern Command), Jammu and Kashmir, India

${ }^{2}$ Graded Specialist in Oral Pathology, Office of Directorate General Dental Services, New Delhi, India

\begin{abstract}
The Keloid is a fibroproliferative anomaly of the cutaneous connective tissue secondary to dysregulation in the skin healing and repair process, occurring in susceptible individuals. It is characterized by excessive collagen and glycoprotein deposition in the dermis following any local irritation, inflammation, burn, incision or injury, thereby leading to a cosmetically unaesthetic, aberrant and exuberant scar formation extending well beyond the boundaries of the original wound. It usually presents as firm nodules, often pruritic and painful, which do not regress spontaneously. The condition presents quite a therapeutic challenge owing to its unpredictably aggressive nature, frequent invasion of adjacent normal dermis, occasional appearance of satellite lesions in nearby non-traumatized tissue and a remarkable tendency for recurrence following removal.
\end{abstract}

Various combinations of Pressure therapy, intralesional steroid therapy and surgery have shown promising results in the treatment of auricular keloids. We report on a young Indian female, aged 19 years, who developed bilateral auricular keloids subsequent to earlobe piercing, which was successfully managed by careful and atraumatic surgical excision alone.

Keywords: Keloid; Hypertrophic scar; Fibroproliferative aberration

\section{Introduction}

The term 'Keloid' was originally described in 1800s as 'Cheloid', which has been derived from the Greek word 'Chele', meaning 'Crab's claw', owing to its appearance [1]. Keloid formation is an aberration in the tissue repair process unique to humans, characterized by the formation of nodular, exuberant growth/s at the site of a dermal injury or a healed / healing wound [2], resulting from an imbalance between anabolic (proliferative) and catabolic (resorptive) phases of the wound reparative process. Unlike the Hypertrophic scar, which remains confined within the boundaries of the original wound, the Keloid is an overgrowth of tissue that extends beyond the borders of the original wound, invading into and around adjacent normal skin. Unlike hypertrophic scars which usually regress after a year or two, keloids typically grow for several years and then become stable or keep growing throughout the lifetime of the patient [3]. Also, although hypertrophic scars respond well to therapy, keloids often do not.

There is a distinctive predilection for certain anatomical locations like the anterior chest, back, shoulders, upper arms, back of the neck, cheeks and ear lobes. They are seen as rubbery or firm nodules or overgrown skin scars, which develop after abrasion, surgery, burns, insect bites, piercing, or similar insults to the skin [4]. They are usually asymptomatic, but may at times present with pain or pruritus.

Keloid treatment is often difficult and frustrating with unpredictable results that are frequently fraught with recurrence. Till date, there exists no comprehensive and definitive treatment strategy that can guarantee a recurrence free outcome. Combination therapy, using surgical excision followed by intradermal steroid instillation and / or pressure therapy or radiotherapy, has been advocated as being more efficacious than single modality therapy [5].

A case of bilateral auricular keloids in an adolescent girl is presented, which was successfully managed by careful surgical excision alone.

\section{Case Report}

A 19 year old female patient reported with the chief complaint of unsightly appearance of her ears caused by painless, brown - black coloured potato-shaped growths on both her ear lobes (Figures 1and 2). History revealed that she had got her ear lobes and middle of the helix on each ear, pierced at a local jeweler's six months ago, following which a small nodule had developed adjacent to the upper piercing (at the distal part of the helix on each side) which had increased slowly, to reach the present size. She also complained of an occasional itching sensation in the affected regions. The growths were circumscribed, ellipsoid in shape, located behind the helix and pinna region of each ear and measured approximately $3 \times 2 \mathrm{~cm}$ (Figures 1 and 3). They had a smooth surface, were of the same colour as that of the adjacent skin, and showed no signs of inflammation or secondary changes. They were pedunculated, attached by a slender stalk to the earlobe/pinna. They were firm, non-compressible, non-fluctuant, non-tender and attached to the overlying skin. On the right ear, in addition to the large keloid there was a smaller nodule just below it (Figure 3C), slightly away from the piercing, probably representing a satellite lesion. History and clinical features pointed to the obvious diagnosis of bilateral auricular keloids secondary to the trauma of the earlobe piercing.

As the patient desired an expedient elimination of the progressively enlarging growths, chiefly for esthetic reasons, it was decided to surgically excise the masses followed by steroid injection if required in case of evidence of recurrence. The patient was taken up for local excision of the keloid on the left earlobe first (Figure 1D-1H). This

*Corresponding author: Jeyaraj P, Classified Specialist (Oral and Maxillofacial Surgery), Deputy Commandant; Officer in Charge Trauma and Rehabilitation, Command Military Dental Centre (Northern Command), Jammu and Kashmir, India, Tel: +91-9596840303; E-mail: jeyarajpriya@yahoo.com

Received November 09, 2017; Accepted November 27, 2017; Published December 04, 2017

Citation: Jeyaraj P, Sehgal S (2017) Surgical Management of a Case of Bilateral Auricular Keloids in an Adolescent Girl. J Cytol Histol 8: 491. doi: 10.4172/21577099.1000491

Copyright: @ 2017 Jeyaraj $P$, et al. This is an open-access article distributed under the terms of the Creative Commons Attribution License, which permits unrestricted use, distribution, and reproduction in any medium, provided the original author and source are credited. 
Citation: Jeyaraj P, Sehgal S (2017) Surgical Management of a Case of Bilateral Auricular Keloids in an Adolescent Girl. J Cytol Histol 8: 491. doi: 10.4172/2157-7099.1000491

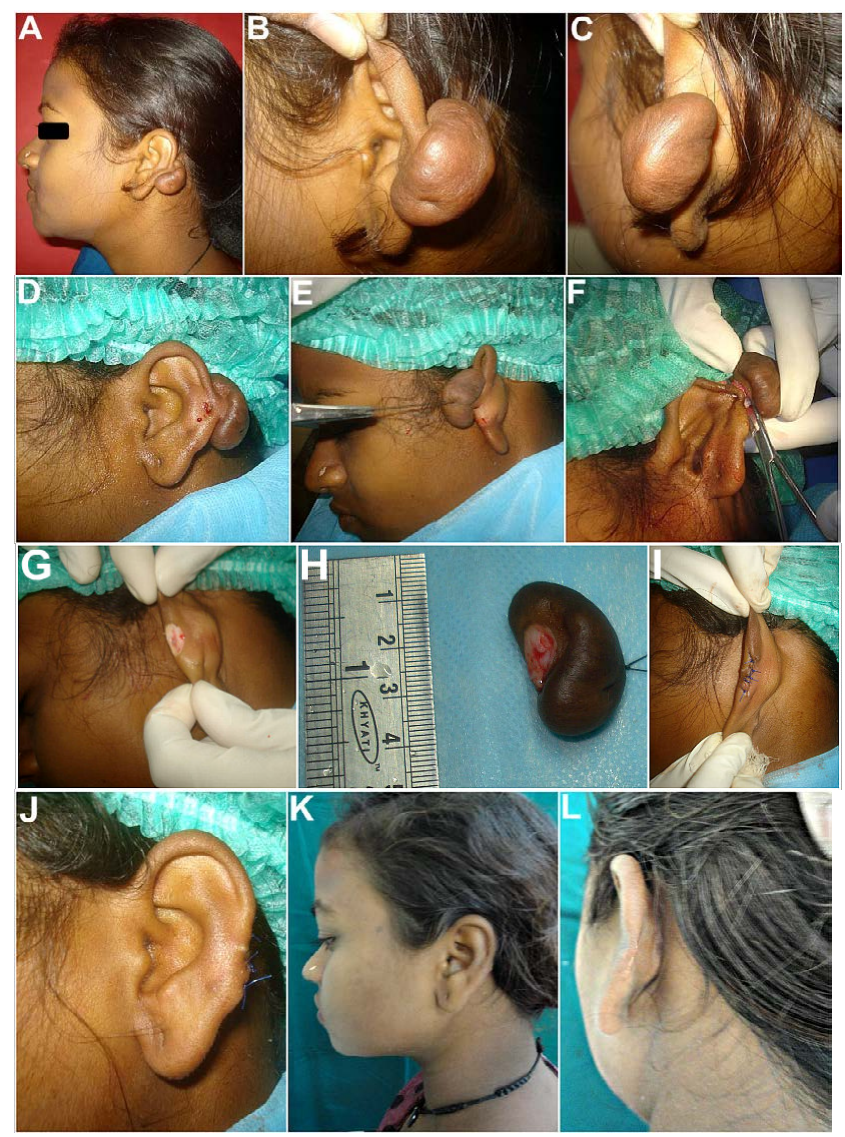

Figure 1: (A-C) A single well circumscribed ellipsoid nodular mass measuring $3 \times 2 \mathrm{~cm}$, with a narrow base, seen behind the helix of the left ear in a 19 year old female patient. (D-H) Local anesthesia infiltrated at the base of the lesion, followed by an atraumatic excision of the mass. (I, J) Wound closed using interrupted Prolene sutures in a tension-free closure. (K, L) Six months post-operative appearance showing no evidence of recurrence.

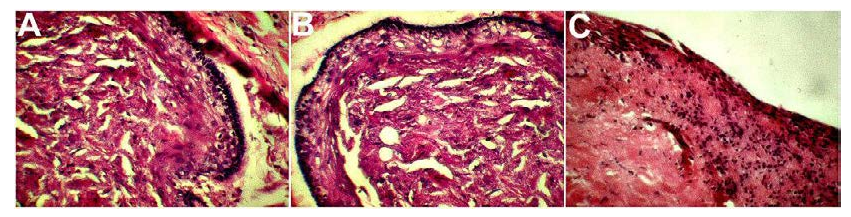

Figure 2: H\&E Section 10X and 40X showed hyper-orthokeratinized stratified squamous epithelium with deep dermal sclerosis and haphazardly arranged, thick bundles of mature collagen fibers which showed a glassy hyalinized appearance, characteristic of the keloid. The ground substance was mucinous, showing relatively few fibroblasts. Small aggregating blood vessels were seen just below the epidermis, and there was seen a moderate degree of perivascular chronic inflammatory cell infiltrate.

was performed taking the precautions of strict asepsis as well as an atraumatic surgery. Tension free skin closure was carried out after careful approximation of the margins, using Prolene sutures, which were removed after ten days (Figure 1I and 1J). Six months follow up showed no recurrence of the lesion (Figure 1K).

The excised mass was sent for histopathological examination, and revealed hyper-orthokeratinized stratified squamous epithelium with deep dermal sclerosis and haphazardly arranged, thick bundles of mature collagen fibers which showed a glassy hyalinized appearance, characteristic of the keloid. The ground substance was mucinous, showing relatively few fibroblasts. Small aggregating blood vessels were seen just below the epidermis, and there was seen a moderate degree of perivascular chronic inflammatory cell infiltrate.

Two months following the excision of the keloid from the left ear, the patient was carefully evaluated and there was found no evidence of recurrence of the keloid. Healing had occurred well and there was no need for intralesional instillation of steroid. The keloid on the right side was excised in a similar fashion (Figure 3 ), and healing proceeded uneventfully, with no recurrence on one year follow up.

\section{Discussion}

Keloids are fibroproliferative anomalies of the cutaneous connective tissue secondary to dysregulation in the skin healing and repair process, occurring in susceptible individuals [1,2]. They are characterized by excessive collagen and glycoprotein deposition in the dermis following any local irritation, inflammation, burn, incision or injury, thereby leading to cosmetically unaesthetic, aberrant and exuberant scar formation extending well beyond the boundaries of the original wound. There occurs an increased fibroblast activity with greater and more sustained extracellular matrix (ECM) deposition and an inhibition of the production of proteases $[3,4]$.

The exuberant scar tissue found in keloids has been attributed to augmented Growth factor activity (transforming growth factor beta and platelet derived growth factor) and alterations in extracellular matrix (fibronectin, hyaluronic acid and biglycan) [4].

Keloids may present as pedunculated or sessile growths which may be uninodular or multinodular, with no particular sex predilection, most commonly affecting patients in the age group of 20-35 years [4]. Keloid formation is approximately 15 times more frequent in highly pigmented ethnic groups like African-Americans and Asians rather than in Caucasians [5]. The pathogenesis of keloid formation is complex

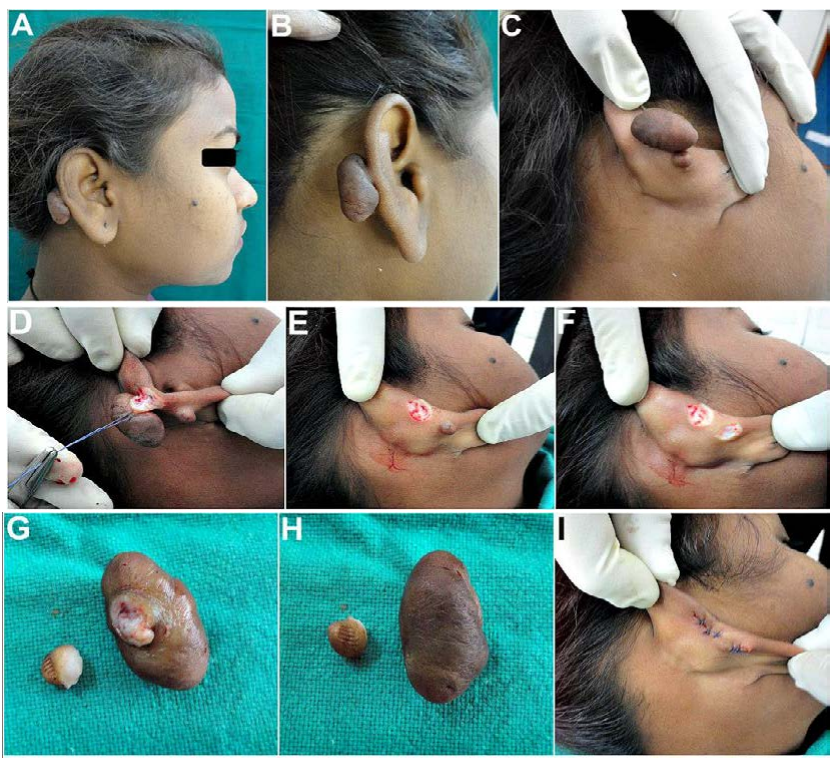

Figure 3: (A-C) Two pedunculated nodular masses behind the right helix excised atraumatically under Local anesthesia. (G, H) Macroscopically, the excised specimen appeared as twonodular, soft, encapsulated masses, the larger ellipsoid one measuring approximately $3 \mathrm{~cm} \times 2 \mathrm{~cm} \times 1 \mathrm{~cm}$, while the smaller mass was spherical measuring $0.5 \mathrm{~cm}$ in diameter. (I) Tension-free closure using interrupted Prolene sutures. 
and involves familial, hormonal, genetic as well as environmental factors [5].

Surgical excision has been associated with a recurrence rate of over $50 \%$ in the past [6]. Factors associated with recurrence following surgical excision include tissue trauma, dead space formation, infection, wound tension and presence of foreign material [7]. Simple total excision of the keloid stimulates additional collagen synthesis, thus prompting a quick recurrence even larger than the initial one, due to which, intramarginal excision of the keloid tissue has been recommended to prevent stimulation of additional collagen synthesis [1].

Due to attempts aimed at overcoming the high rate of recurrence following surgical excision, a plethora of management modalities and a variety of therapies exist in literature for this challenging, troublesome, persistent, and often recurrent condition. Keloids are not only aesthetically ungainly, but also capable of reaching sizes that can interfere with function as well. Management modalities include pressure therapy, intralesional steroid injection, radiotherapy, brachytherapy, cryotherapy, silicone gel sheeting, flavanoids as well as laser removal [6]. Less commonly employed modalities include topical imiquimod, intralesional interferon and anti-metabolites like 5 -fluorouracil and bleomycin $[7,8]$. However, none of these treatments can be considered as the gold standard or an exclusive management modality. Combination therapies have met with more success in the past as compared to single modality therapy.

Ear lobe keloids have been managed by surgical excision and pressure therapy using methyl methacrylate pressure stents [9] and pressure clips [10]. These pressure devices modulate collagen metabolism by producing a hypoxic environment by application of pressure exceeding the capillary pressure, thus causing fibroblastic degradation and necrosis. Application of a controlled and continuous pressure of $24-30 \mathrm{~mm} \mathrm{Hg}$, by an optimally aligned custom made appliance has been considered ideal [11,12]. A drawback of this treatment modality is a heavy reliance on patient compliance. Hence for a successful outcome or even for a partial reduction in the size of the outgrowth, the patient must be motivated to wear the appliance for a reasonable length of time.

Another treatment protocol for auricular keloids involves intralesional steroid injection, most commonly Triamcinolone, and pressure therapy to reduce the size of the mass followed by surgical excision, which is again followed by steroid injection and pressure therapy for a period of 6 to 12 months at the site in order to reduce the chances of recurrence $[4,13]$. Steroids act by inhibiting the Alpha-2macroglobulin, which when active, normally inhibits collagenase. Thus the use of topical or intralesional corticosteroids, enable collagenase to be active, thus promoting collagen degeneration. Commonly reported side effects include hypopigmentation, skin atrophy, telangiectasia, etc. $[14,15]$. Patient compliance is again an issue here as following surgical excision, the patient very often does not return for the follow up therapy.

Radiotherapy has been used as monotherapy or as an adjuvant after surgical excision of keloids. Among the adverse effects of radiotherapy are pigmentary alterations, radiation dermatitis, and potential carcinogenesis [16]. A dosage of 300 rads (3 Gy) for 5 courses or 500 rads $(5 \mathrm{~Gy}$ ) for 3 course starting immediately after surgery when the fibroblasts are proliferating has shown some positive results $[17,18]$.

Laser and Cryotherapy are also modalities of surgical therapy that are sometimes used in the treatment of keloids [14]. Cryosurgery as a monotherapy involves two courses of 15-20 second freeze-thaw cycles every three weeks. The rapid repeated cooling and rewarming of tissue leads to cell death and tissue sloughing, while at the same time, also has a direct beneficial effect on keloid collagen, resulting in improved collagen bundle organization. Local hyper- or hypopigmentation are possible side effects [14].

Intralesional Interferons have been of some use in the management of keloids by virtue of their ability to interfere with collagen synthesis and fibroblast proliferation, thereby producing an anti-fibrotic effect by antagonizing the action of transforming growth factor (TGF) beta and histamine [19].

\section{Conclusion}

The outcome of an altered tissue response to a physical stimulus results in the formation of keloids, the pathophysiology of which is till date not clearly understood. There exists no ideal, comprehensive or definitive treatment modality which can ensure a successful management with non-recurrence of the keloids; however, literature suggests that a combination of two or more treatment modalities has a better prognosis than a single modality therapy. The case presented highlights that even a simple intra-marginal surgical excision alone can provide the desired results, provided a diligent and atraumatic surgical technique followed by tension-free closure is employed and a long term post-op follow-up is carried out.

\section{Compliance with Ethical Standards}

\section{Disclosure of potential and conflicts of interest}

The author of this article has not received any research grant, remuneration, or speaker honorarium from any company or committee whatsoever, and neither owns any stock in any company. The author declares that she does not have any conflict of interest.

Research involving human participants and /or animals: All procedures performed on the patients (human participants) involved were in accordance with the ethical standards of the institution and/ or national research committee, as well as with the 1964 Helsinki declaration and its later amendments and comparable ethical standards.

Ethical approval: This article does not contain any new studies with human participants or animals performed by the author.

\section{Informed consent}

Informed consent was obtained from all the individual participants in this study.

\section{Funding}

This study was not funded by any organization/society.

\section{References}

1. Hunasgi S, Koneru A, Vanishree M (2013) Keloid - A case report and review of pathophysiology and differences between kelod and hypertrophic scars Journal of Oral and Maxillofacial Patholgy 17: 116-120.s

2. Robles DT, Berg D (2007) Abnormal wound healing: Keloids. Clin Dermatol 25: 26-32.

3. Kang N, Sivakumar B, Sanders R (2006) Intra-lesional injections of collagenase are ineffective in the treatment of keloids and hypertrophic scars. J Plastic Reconstr Aesthet Surg 59: 693-699.

4. Bala S, Sikka N, Kannupriya (2016) Comprehensive management of bilatera auricular keloids: A case report. Sch J Med Case Rep 4: 909-912.

5. Moshref SS, Mufti ST (2010) Kelod and Hypertrophic scars: Comparative histopathological and immunohistochemical study. Med Sci 17: 3-22. 
Citation: Jeyaraj P, Sehgal S (2017) Surgical Management of a Case of Bilateral Auricular Keloids in an Adolescent Girl. J Cytol Histol 8: 491. doi: 10.4172/2157-7099.1000491

6. Gauglitz GG, Korting HC, Pavicic T (2011) Hypertrophic scarring and Keloids: Pathomechanisms and current and emerging treatment strategies. Mol Med 17: $113-125$

7. Wolfram D, Tzankov A (2009) Hypertrophic scars and keloids: A review of their pathophysiology, risk factors, and therapeutic management. Dermatol Surg 35: 175-181.

8. Ragoowansi R, Cornes PGS, Powell BW (2001) Ear lobe keloids: treatment by a protocol of surgical excision and immediate adjuvant radiotherapy. $\mathrm{Br} \mathrm{J}$ Plas Surg 54: 504-508.

9. Sela M, Taicher S (1984) Prosthetic treatment of earlobe keloids. J Prosthet Dent 52: 417-418.

10. Vachiramon A, Bamber MA (2004) A U-loop pressure clip for earlobe keloid. J Prosthet Dent 92: 389-391

11. Chrisostomidis C, Konofaos Vasilopapoulou A (2008) Management of external ear keloids using form-pressure therapy. Clin Exp Dermatol 33: 273-275.

12. Mutalik S (2005) Treatment of keloids and hypertrophic scars. Indian J Dermato Venereol Leprol 71:3-8.
13. Lawrence WT (1991) In search of the optimal treatment of keloids: report of a series and a review of the literature. Ann Plast Surg 27: 164-178.

14. Rusciani L, Paradisi A, Alfano C (2006) Cryotherapy in the treatment of keloids. J Drugs Dermatol 5: 591-595.

15. Alster TS, Tanzi EL (2003) Hypertrophic scars and keloids: etiology and management. Am J ClinDermatol 4: 235-243.

16. Van de Kar El, Kreulen M, van Zuijlen PP (2007) The results of surgical excision and adjuvant irradiation for therapy-resistant keloids: A prospective clinical outcome study. Plast Reconstr Surg 119: 2248-2254.

17. Sanders KW, Gage-White L, Stucker F (2005) Topical Mitocin-C in the prevention of keloid scar recurrence. J Arch Facial Plast Surg 7: 172-175.

18. Park TH, Seo SW, Kim JK, Chang CH (2012) Clinicalcharacteristics of facial keloids treated withsurgical excision followed by intra- andpostoperative intralesional steroid injections. Aesthetic Plast Surg 36: 169-173.

19. Brown JJ, Bayat A (2009) Genetic susceptibility to raised dermal scarring. $\mathrm{Br}$ J Dermatol 161: 8-18. 